\title{
Behavioral Activation and Behavioral Inhibition Predict Perfectionism
}

\author{
Charles Palmer Mautz, Robert Wallace Hill, Timothy J. Hueslman, Doris G. Bazzini
}

Department of Psychology, Appalachian State University, Boone, USA

Email address:

hillrw@appstate.edu (R. W. Hill)

To cite this article:

Charles Palmer Mautz, Robert Wallace Hill, Timothy J. Hueslman, Doris G. Bazzini. Behavioral Activation and Behavioral Inhibition Predict Perfectionism. Psychology and Behavioral Sciences. Vol. 6, No. 4, 2017, pp. 59-64. doi: 10.11648/j.pbs.20170604.13

Received: June 2, 2017; Accepted: June 27, 2017; Published: July 24, 2017

\begin{abstract}
This investigation assessed of the ability of Reinforcement Sensitivity Theory (RST), comprising the Behavioral Activation System (BAS), and Behavioral Inhibition System (BIS) to predict perfectionism. A total of 508 community participants were acquired using MTurk and completed measures of personal standards and self-evaluative perfectionism, as well as BIS and BAS. Regression analyses revealed that BAS sensitivity successfully predicted personal standards perfectionism (and to a lesser extent self-evaluative perfectionism), while BIS sensitivity successfully predicted self-evaluative perfectionism (and to a lesser extent personal standards perfectionism). BAS and BIS were robust predictors in their respective regression models, suggesting that these important perfectionism factors are successfully and jointly predicted by the BAS/BIS pre-dispositional motivation constructs.
\end{abstract}

Keywords: Perfectionism, Reinforcement Sensitivity Theory, Behavioral Activation System, BAS, Behavioral Inhibition System, BIS

\section{Introduction}

Reinforcement sensitivity theory (RST) proposes that certain neuropsychological processes partially govern responses to environmental stimuli, forming the basis for individual personality dispositions $[7,11,22]$. Perfectionism has been described as a multidimensional set of behaviors and beliefs, characterized by a personal strivings factor and by a self-evaluative concerns and worries factor [20]. Using the theory of RST to explain internal responses to the perception of reward and threat related to striving for excellence and flawlessness, the role of RST mechanisms in providing partial biological underpinnings to perfectionistic personality characteristics was investigated.

\subsection{Reinforcement Sensitivity Theory}

Reinforcement sensitivity theory is a broad attempt to relate neuropsychological regulation of human behavior to individual personality [7] Initially proposed in 1970 by Jeffrey Gray, RST has been modified several times over the last four decades. Revised RST (RST-R) proposes that individual differences are related to constitutionally determined individual states, and in turn, overarching traits of personality [7]. Essentially, RST-R suggests that hard-wired brain activation tendencies influence one's interpretation of and reaction to environmental stimuli [7]. RST-R does not suggest that neuropsychological processes completely control one's reaction to a stimulus, as these underlying processes instead serve to influence the relationship between environmental stimuli (input) and reactions (output) in individual behavior. The reactions are internal processes, not necessarily the physical behavior exhibited [7].

According to RST-R, strong Behavioral Activation System (BAS) tendencies indicate an individuals' predisposition toward welcoming and approaching conditioned positive outcomes, or those positive outcomes that they have associated with personal experiences. Those displaying strong BAS approach tendencies show strong neurological motives to perform, achieve success, and strive to attain the positive outcomes. High BAS sensitivity has been described as seeking incentives and rewards through pursing lofty goals [15] which also appears to be true of individuals high in personal strivings perfectionism. Strong Behavioral Inhibition System (BIS) tendencies reflect risk assessment and anxiety in the face of goal conflict, including hesitation, 
and fear, influencing approach versus avoidance behavior, when confronted with potential punishments. These punishments can vary in specifics, but in general are perceived situations in which a loss, injury, or other harmful situation is at hand. The Fight-Flight-Freeze System (FFFS) system responds to immediate threats, influencing avoidance behavior, and was distinguished from BIS in later visions of RST. FFS is theorized to work alongside the BIS, allowing one to develop a predisposition towards a particular form of avoiding or alleviating a negative outcome [7]. FFS was not hypothesized to play a significant role with perfectionism for this study, nor the others reviewed here, and is also included in the BIS scale in the widely used Carver and White (1994) BIS/BAS scales.

RST-R purports that the mechanisms at play are neurological, which allow only for internal responses to stimuli. As such, these responses do not entirely dictate behavior; rather, they influence one's interpretation of stimuli and theoretically only predispose behavior. In other words, the RST-R mechanisms can set a stage for behavior without one's conscious awareness, but other factors can also have meaningful influence on behaviors, thoughts, and emotional responses to events [7].

\subsection{Perfectionism}

After years of research characterizing perfectionism as a unidimensional construct, two teams of researchers published multidimensional perfectionism scales (MPS) in 1990 [9, 12]. The Frost and colleagues MPS measure described perfectionism using 6 scales: personal standards, organization, concern over mistakes, doubts about actions, parental expectations, and parental criticism. The Hewitt and Flett (1991) MPS provided 3 scales: self-oriented, otheroriented, and socially- prescribed perfectionism. Following this research establishing the multidimensionality of perfectionism, factor analytic studies of the MPS instruments revealed a two-factor construct, comprising factors that can be labeled as "perfectionistic strivings" and "self-evaluative concerns" [20]. Personal strivings perfectionism describes tendencies to strive for success, pursue lofty goals or expectations, engage in extensive and effective preparation, tendencies to persevere, to be diligent, organized, resilient, and to endure through struggle. Self-evaluative perfectionism describes tendencies to worry about previous or potential future mistakes, to ruminate, to perceive significant external pressure, or to be concerned with comparisons between one's achievements and perceived expectations [20]. Both dimensions clearly are aimed towards attaining perfect behavior but in different ways.

For both perfectionistic strivings and self-evaluative dimensions, perfectionism is a set of personality traits influencing thoughts, beliefs, behavior, and even pathologies. Positive outcomes including life and task satisfaction, lower stress levels, active coping styles, positive affect, higher conscientiousness, and higher levels of achievement are commonly associated with perfectionistic strivings perfectionism [4, 20, 21]. Conversely, negative outcomes including depression, anxiety, stress, lower self-esteem, and increased performance anxiety are associated with selfevaluative perfectionism $[1,20,21]$.

\subsection{RST and Perfectionism}

To the extent that RST-R has value and primacy in explaining behaviors, RST-R variance was expected to explain perfectionistic traits. In particular, it was expected that given high sensitivity to BAS, an individual inclined to feel excited about success in the face of a conditioned positive stimulus, and thus might be expected to show striving for excellence, have high expectations for achievement, and engage in extensive preparation, all traits of perfectionistic strivings perfectionism. Conversely, sensitivity to negative outcomes, or potentially negative outcomes, described by the BIS, might predict self-evaluative perfectionism, as high BIS suggests that an individual is nervous, anxious or intimidated in the face of a potentially upsetting, disappointing or frustrating stimulus. An individual with high BIS might exhibit concern and rumination over past and future mistakes, fear failure and fear negative evaluation from others, which are characteristic of self-evaluative perfectionism.

Further, the neuropsychological predispositions described by BAS and BIS were expected to precede the behavioral tendencies described by perfectionistic strivings and selfevaluative perfectionism, respectively, as the former are innate neurological predispositions and the latter personality traits. Therefore, BAS tendencies to approach reward may be antecedent to perfectionistic behaviors geared towards success, including perfectionistic strivings perfectionism. BIS tendencies to avoid negativity or loss could precede perfectionistic concerns related to failure, and negative selfappraisal, including self-evaluative perfectionism.

In a previous investigation of RST-R, perfectionism and worry, Chang and colleagues (2007) found mixed correlations between BIS and BAS and various indices of perfectionistic strivings and self-evaluative perfectionism. They used a multidimensional measure of perfectionism [9] and the BIS/BAS scales developed by Carver and White (1994), and their results revealed correlations between BAS scales and both perfectionistic strivings and self-evaluative perfectionism, and also correlations between BIS and indices of both perfectionistic strivings and self-evaluative perfectionism. They did report that a BIS disposition was most useful in predicting worry, along with primarily selfevaluative perfectionism dimensions, which accounted for significant variance after BIS was removed. Another team of researchers assessing RST-R using the Carver and White (1994) BIS/BAS scales, parenting, and maladaptive perfectionism, found that BIS was positively associated with self-evaluative perfectionism indices, and not BAS [23]. However, that investigation did not report using a measure of perfectionistic strivings perfectionism, and they selected only 2 subscales (Doubts About Actions, and Concern Over Mistakes) from the Frost and colleagues (1990) perfectionism measure to represent self-evaluative 
perfectionism. Similarly, Rasmussen, Elliot, and Carter (2012) investigated the role of RST-R and sociallyprescribed perfectionism (an index of self-evaluative perfectionism) in predicting suicidal thinking, and found BIS but not BAS associated with socially-prescribed perfectionism (as expected). They also noted negative associations between BAS and suicidal thinking, hopelessness and depression. They did not report any findings for perfectionistic strivings perfectionism. Randles and colleagues (2010) also assessed the relationship between BAS, BIS and perfectionism. Essentially, using the threefactor model of perfectionism from Hewitt and Flett's (1991) Multidimensional Perfectionism Scales, Randles and colleagues found that BAS and BIS sensitivity, assessed by the Carver and White (1994) BIS/BAS scales, were both associated with high perfectionistic strivings (self-oriented perfectionism). They further found that BIS was also associated with one's perceptions of high expectations from others (socially-prescribed perfectionism) as well as trait rumination [16]. They also documented a modest correlation between socially-prescribed perfectionism and BAS Reward.

In summary, these previous investigations provided mixed findings regarding the relationship between BAS, BIS and the perfectionistic strivings, and self-evaluative perfectionism factors, and thus, invited follow-up investigation. The current study was conducted to further clarify the ability of the dimensions of BAS and BIS to predict perfectionistic strivings and self-evaluative perfectionism respectively, and to further consider the etiology of perfectionism as partially neuropsychologically driven by RST-R. The Carver and White (1994) measure of BSI/BAS was selected, despite of some documented concerns with this measure of RST/RST-R [18], as this has been the most widely used measure, particularly in the literature relevant to perfectionism.

The Perfectionism Inventory, rather than the Multidimensional Perfectionism Scales (MPS), was selected to measure perfectionistic strivings and self-evaluative perfectionism. The Perfectionism Inventory provides clear examples of perfectionistic strivings and self-evaluative perfectionism indices, described during scale construction [13], which are less clearly derived from the MPS scales. The 3 MPS scales by Hewitt and Flett (1990) were developed prior to factor-analytic research revealing the utility of a 2 factor perfectionism model $[8,20]$.

Sensitivity to BAS was hypothesized to positively associate with perfectionistic strivings perfectionism and and also sensitivity to BIS would positively associate with selfevaluative perfectionism. However, as BAS and BIS are not mutually exclusive, potential associations between BIS and perfectionistic strivings perfectionism and between BAS and self-evaluative perfectionism were also expected. Given the mixed findings of previous investigations of these constructs this investigation expected to add to the research literature with an additional analysis of associations, and the assessment of the value of RST-R in predicting the 2-factor model of perfectionism.

\section{Method}

\subsection{Participants and Procedure}

Participants for this investigation were drawn from Amazon's Mechanical Turk (MTurk), a web-service intended to provide human feedback on various tasks. MTurk data have been shown to represent the US population well, be diverse, provide quality participation despite compensation rates, and be at least as reliable as data from various other sources [2]. The task was opened on Mturk only for users claiming the United States as their nation of origin. Of the 557 completed cases, eight cases were removed for having completed the task twice. Further, 41 cases were removed because of invalid responding as identified by endorsement of three or more items on the Infrequency Scale for Personality Measures [ISPM; 14] leaving 508 cases in the data set.

Of the 508 cases, $65 \%$ (335 cases) were female, $34 \%$ (153 cases) were male, and the average respondent age was 32.4 years, with a standard deviation of 15.6 years and a range of 18 to 81 years. Descriptive statistics regarding respondent annual household income, ethnic background, and highest level of education achieved can be found in Table 1 .

\subsection{Measures}

Perfectionism Inventory (PI). The PI is a 59-item measure comprising eight subscales with internal consistency reliability ranging from .83 to .91 . The Conscientious Perfectionism composite score is derived from the sum of the following scales: Organization, Striving for Excellence, Planfulness, and High Standards for Others. The SelfEvaluative

Perfectionism composite score is derived from the sum of the following scales: Concern over Mistakes, Need for Approval, Rumination, and Perceived Parental Pressure. In this investigation the Conscientious Perfectionism composite was used to assess perfectionistic strivings, and the SelfEvaluative Perfectionism composite was used to assess perfectionistic concerns. Hill and colleagues (2004) report that the PI has good convergent validity with other measures of perfectionism. All PI scales have good variability and clear unidimensional structures, as reflected in exploratory principal components analyses, confirmatory factor analysis, and internal consistency. Test-retest correlations for the eight PI scales ranged from .71 to .91 over four to five weeks [13]. An example item from the conscientious subscale organization is "I am well-organized." An example item from the self-evaluative subscale perceived parental pressure is "My parents hold me to high standards" [13].

BIS/BAS Scales. There does not appear to be a widely agreed upon best measure for RST-R, particularly for BAS [18]. The most widely used measure of RST, developed by Carver and White (1994) was used for this study. The BIS/BAS Scales have 20 items measuring: BIS, assessing reactivity to negative or aversive stimuli as a single construct, and BAS as three subscales: Drive, Funseeking, and Reward 
Responsiveness. Drive describes a persistent pursuit of goals, Funseeking a spontaneous or spur of the moment pursuit of positive outcomes, and Reward Responsiveness a positive anticipation of outcomes. The Carver and White (1994) conceptualization of BIS encompasses both BIS and fight-orflight system sensitivities, consistent with the original reinforcement sensitivity theory model, and has been used in many studies assessing RST-R. Data collected with the BIS/BAS Scales have shown adequate internal consistency, with Cronbach's alphas for the three BAS subscales ranging from .66 to .76, and .74 for the BIS subscale [3]. Data collected with this measure have also demonstrated an adequate level of construct validity for behavioral activation and inhibition. An example item from the BIS scale is "I worry about making mistakes." An example item from the BAS drive scale is "I go out of my way to get things I want." [3].

The Infrequency Scale for Personality Measures (ISPM). The ISPM is a 13-item scale that is embedded among other measures to ensure the valid responding of participants. An endorsement of any item of the measure is extremely unlikely (e.g. "I cannot remember when I talked with someone who wore glasses") and indicates a potentially invalid response style. To maintain consistency with how the ISPM has been used in previous studies, endorsement of three or more items on the ISPM indicates invalid responding and excluded the participant from analyses [14].

\section{Results}

Means, standard deviations, zero-order correlations, and internal consistency reliabilities for study scales are presented in Table 1. It is notable, and consistent with the previous literature, that perfectionistic strivings perfectionism and self-evaluative perfectionism were positively correlated, $r=.49$. However, contrary to expectations, perfectionistic strivings perfectionism and self-evaluative perfectionism were each positively correlated with both BAS and BIS (except perfectionistic strivings perfectionism was not correlated with BAS-Funseeking). Self-evaluative perfectionism did have a stronger relationship with BIS ( $r$ $=.67$ ) than with BAS (mean $r=.12$ ), consistent with the logic of the hypotheses.

Table 1. Descriptive statistics and correlation coefficients for all study variables.

\begin{tabular}{|c|c|c|c|c|c|c|}
\hline Variable & PS-P & SE-P & BAS-D & BAS-FS & BAS-RR & BIS \\
\hline PS Perfectionism & .92 & & & & & \\
\hline SE Perfectionism & $.49 * * *$ & .95 & & & & \\
\hline BAS-D & $.35 * * *$ & $.19 * * *$ & .88 & & & \\
\hline BAS-FS & -.05 & $.08 *$ & $.26 * * *$ & .10 & & \\
\hline BAS-RR & $.22 * * *$ & $.09 *$ & $.43 * * *$ & $.13 * *$ & .83 & \\
\hline BIS & $.32 * * *$ & $.67 * * *$ & $.10 *$ & $-.08 *$ & $.26 * * *$ & .81 \\
\hline Mean & 14.08 & 12.36 & 11.46 & 10.34 & 17.90 & 20.76 \\
\hline
\end{tabular}

Notes. Values in italics on the main diagonal are Cronbach's coefficient alpha. PS-P $=$ Personal Strivings Perfectionism. SE-P $=$ Self-Evaluative. BAS-D $=$ Behavioral Activation System-Drive. BAS-F = Behavioral Activation System-Fun Seeking. BAS-RR = Behavioral Activation System-Reward Responsiveness. BIS = Behavioral Inhibition System. $N=508 . * p<.05 . * * p<.01 . * * * p<.001$.

To more completely examine the relationships between perfectionism and RST-R, separate standard multiple regressions for perfectionistic strivings perfectionism and self-evaluative perfectionism were conducted, using BAS and BIS as predictors (see Tables 2 and 3 ).

Table 2. Standard multiple regression of Personal Strivings Perfectionism on BAS and BIS.

\begin{tabular}{llllll}
\hline Predictor & $\boldsymbol{B}$ & $\boldsymbol{\beta}$ & $\boldsymbol{t}$ & $\boldsymbol{p}$ & $\boldsymbol{r}$ \\
\hline BAS-D & .30 & .35 & 7.81 & .000 & $.35^{* * * *}$ \\
BAS-F & -.17 & -.12 & -2.99 & .003 & .05 \\
BAS-RR & .02 & .02 & .34 & .738 & $.22^{* * *}$ \\
BIS & .15 & .27 & 6.55 & .000 & $.32^{* * *}$ \\
Constant & 9.02 & & & & $F_{4,503}=34.98, p<.001$ \\
$R=.47$ & $R^{2}=.22$ & Adj $R^{2}=.21$ & & \\
\hline
\end{tabular}

Notes. BAS-D = Behavioral Activation System-Drive. BAS-FS = Behavioral Activation System-Fun Seeking. BAS-RR = Behavioral Activation SystemReward Responsiveness. BIS $=$ Behavioral Inhibition System. ${ }^{* * *} p<.001$.

Table 3. Standard multiple regression of Self-Evaluative Perfectionism on BAS and BIS.

\begin{tabular}{lllll}
\hline Predictor & $\boldsymbol{B}$ & $\boldsymbol{\beta}$ & $\boldsymbol{t}$ & $\boldsymbol{p}$ \\
\hline BAS-D & .19 & .17 & 4.77 & .000 \\
BAS-FS & .21 & .12 & 3.52 & .000 \\
BAS-RR & -.26 & -.18 & -5.11 & .000 \\
BIS & .52 & .71 & 21.89 & .000 \\
Constant & 1.87 & & & $.09^{* * *}$ \\
$R=.71$ & $R^{2}=.51$ & $\operatorname{Adj} R^{2}=.50$ & & $F_{4,503}=129.22, p<.001$ \\
\hline
\end{tabular}

Notes. BAS-D = Behavioral Activation System-Drive. BAS-FS = Behavioral Activation System-Fun Seeking. BAS-RR $=$ Behavioral Activation SystemReward Responsiveness. BIS = Behavioral Inhibition System. ${ }^{*} p<.05 . * * * p<.001$. 
For personal striving perfectionism, the BAS variables were expected to be stronger predictors than BIS, even if the zero-order correlations were not consistent with this prediction. The multiple regression indicates that the set of predictors accounts for more than $20 \%$ of the variance in personal striving perfectionism $\left(R^{2}=.22, F_{4,503}=34.98, p\right.$ $<.001$ ), with BAS Drive, BAS Funseeking, and BIS all making significant contributions to the regression equation; BAS Drive $(\beta=.35)$ and BIS $(\beta=.27)$ were the strongest contributors.

For self-evaluative perfectionism, BIS was expected to be a stronger predictor than the BAS variables. Again, the zeroorder correlations were not consistent with this prediction. The multiple regression indicated that the set of predictors accounted for more than $50 \%$ of the variance in personal striving perfectionism $\left(R^{2}=.51, F_{4,503}=129.22, p<.001\right)$, with all four predictors making significant contributions to the regression equation; BIS $(\beta=.71)$ was clearly the strongest contributor (consistent with the zero-order correlations).

\section{Conclusions}

This investigation did lend support to the expectation that BAS sensitivity would predict perfectionistic strivings perfectionism, and BIS would predict self-evaluative perfectionism. However, the results also reflected considerable shared variance in the value of both BAS and BIS predicting particularly perfectionistic strivings perfectionism, with each RST-R dimension contributing meaningfully.

The regression results indicated that BAS Drive most positively predicts perfectionistic strivings perfectionism, suggesting that the more an individual displays a basic neuropsychological urge to strive for positivity with effort, the more likely they may be to display the achievement oriented, organized and planful high perfectionistic strivings perfectionism. BAS Funseeking may be counterproductive to perfectionistic strivings perfectionism, as the regression results indicate lower tendencies to spontaneously engage in momentary positive experience may be indicative of a higher perfectionistic strivings perfectionism. The Funseeking subscale also demonstrated poor inter-item consistency for this sample indicating more error in analyses using this subscale than other measures.

BIS also was a significant predictor of perfectionistic strivings perfectionism, as found by Chang and colleagues (2007) and Randles and colleagues (2010), indicating that the basic function of assessing risk and avoiding negative stimuli appears to significantly contribute to the tendency toward high achievement. That both BAS and BIS predict perfectionistic strivings perfectionism, evidenced in several investigations now, indicate that both approach and avoidance dispositions contribute to the motivational basis of the personality features of striving for excellence, planfulness, organization and having high standards for others performance.
BIS more robustly predicted self-evaluative perfectionism, accounting for twice the variance of the perfectionistic strivings perfectionism dimension. This was perhaps the most compelling finding of the investigation, in that BIS sensitivity is successful in predicting perfectionistic tendencies to worry, feel anxiety about mistakes, feel pressure from external sources, and ruminate over past behavior. All these personality characteristics, definitive of self-evaluative perfectionism, are manifestations of anxiety or worry over experiencing negative stimuli or outcomes. This relationship suggests that a significant portion of the behavioral and cognitive tendencies of self-evaluative perfectionism may be derived from an antecedent disposition to experience anxiety or hesitation in the presence of negative stimuli. While BAS-drive contributed modest predictive variance as well, the role of BIS appears dominant, consistent with expectations, and RST-R. Subsequent to data collection for this investigation Stoeber and Corr (2015) assessed perfectionism with a new measure of RST-R reporting that perfectionistic strivings perfectionism was positively associated with BAS, BIS and FFFS; selfevaluative perfectionism was associated with BIS, BAS impulsiveness, and negatively with BAS goal drive persistence, but not associated with BAS reward interest or BAS reward reactivity. These results were consistent with the findings from this investigation, with some greater specificity provided by the new RST-R scales used by Stoeber and Corr.

That both BAS and BIS dimension are involved in predicting both perfectionistic strivings and self-evaluative perfectionism, may be supportive of a view of perfectionism as involving competing appetitive versus aversive drives, like many other complex human personality traits that are related to social behaviors. Corr (2001), in his joint subsystems hypothesis, suggested that BAS and BIS might interact in response to an array of both appetitive and aversive stimuli, with BIS detecting conflict as well as punishment, and competing with BAS to influence behavior. Corr (2001) further describes evidence that aversive motivation often carries more weight than appetitive motivation, consistent with these results with the power of BIS over BAS in predicting perfectionism. This hypothesis was also voiced by Rasmussen and colleagues (2012) in their explanation of the interaction of BAS/BIS in predicting suicidal distress.

In summary, these findings suggest that pre-dispositional tendencies in response to stimuli, both approach oriented (BAS) and avoidance oriented (BIS), robustly predict perfectionistic personality, with particular success in predicting self-evaluative perfectionism. These results indicate that RST-R, which suggests biologically driven motivation dispositions, usefully predict perfectionistic personality trait dimensions, and thus inevitably influences achievement, and also the vulnerability to the various indices of psychopathology associated with perfectionism.

Future research might seek to assess the role of BAS/BIS and perfectionism in predicting specific achievement indices (e.g. school performance, sport performance), adaptive 
coping indices (e.g. life satisfaction, stress levels) as well as psychopathology, potentially assessing the possible mediating or moderating role that perfectionism might play in vulnerability to depression, anxiety and eating disorders for example. This investigation was limited by using only one measure of perfectionism, and one measure of RST/RSTR. Future investigations might utilize other measures of perfectionism, and of RST/RST-R, to provide multiple methods of defining the constructs, and either replicating or clarifying the measurement of the 2 factor model of perfectionism, and of BAS/BIS, and clarifying the role of RST-R with perfectionism.

\section{References}

[1] Bieling, P. J., Summerfeldt, L. J. Israeli, A. L. \& Antony, M. M. (2004). Perfectionism as an explanatory construct in comorbidity of axis I disorders. Journal of Psychopathology and Behavioral Assessment, 26(3), 193-201.

[2] Buhrmester, M. D., Kwang, T., \& Gosling, S. D. (2011). Amazon's mechanical Turk: A new source of inexpensive, yet high-quality data? Perspectives on Psychological Science, 6, 3-5. doi: $10.1177 / 1745691610393980$

[3] Carver, C. S., \& White, T. L. (1994). Behavioral inhibition, behavioral activation, and affective responses to impending reward and punishment: The BIS/BAS scales. Journal of Personality and Social Psychology, 67, 319-333. doi: 10.1037/0022-3514.67.2.319

[4] Chang, E. C., Watkins, A., \& Banks, K. H. (2004). How Adaptive and Maladaptive Perfectionism Relate to Positive and Negative Psychological Functioning: Testing a StressMediation Model in Black and White Female College Students. Journal of Counseling Psychology, 51(1), 93-102.

[5] Chang, E. C., Zumberg, K. M., Sanna, L. J., Girz, L. P., Kade, A. M., Shair, S. R., Hermann, N. B., \& Srivastava, K. (2007). Relationship between perfectionism and domains of worry in a college student population: Considering the role of BIS/BAS motives. Personality and Individual Differences, 43(4), 925936.

[6] Corr, P. J. (2001). Testing problems in J. A. Gray's personality theory: A commentary on Matthews, Gilliland (1999). Personality and Individual Differences, 30, 333-352.

[7] Corr, P. J. (2008). The reinforcement sensitivity theory of personality. New York, NY: Cambridge University Press.

[8] Frost, R. O., Heimberg, R. G., Holt, C. S., Mattia, J. I., \& Newbauer, A. L. (1993). A comparison of two measures of perfectionism. Personality and Individual Differences, 14, 119-126. doi: 10.1016/0191-8869(93)90181-2

[9] Frost, R. O., Marten, P., Lahart, C., \& Rosenblate, R. (1990). The dimensions of perfectionism. Cognitive Therapy and Research, 14, 449-468. doi: 10.1007/BF01172967

[10] Gray, J. A. (1970). The psychophysiological basis of introversion-extroversion. Behavior and Research Therapy, 8 , 249-266. doi: 10.1016/0005-7967(70)90069-0
[11] Gray, J. A., \& McNaughton, N. (2000). The neuropsychology of anxiety: An enquiry into the functions of the septohippocampal system (2nd ed.). Oxford, UK: Oxford University Press.

[12] Hewitt, P. L., \& Flett, G. L. (1991). Perfectionism in the self and social contexts: Conceptualization, assessment and association with psychopathology. Journal of Personality and Social Psychology, 60, 456-470.

[13] Hill, R. W., Huelsman, T. J., Furr, M. R., Kibler, J., Vicente, B. B., \& Kennedy, C. (2004). A new measure of perfectionism: The perfectionism inventory. Journal of Personality Assessment, 82, 80-91. doi: 10.1207/s15327752jpa8201_13

[14] Hundt, N. E., Kimbrel, N. A., Mitchell, J. T., \& Nelson-Gray, R. O. (2008). High BAS, but not low BIS, predicts externalizing symptoms in adults. Personality and Individual Differences, 44, 565-575. doi: 10.1016/j.bbr.2011.03.031

[15] Johnson, S. L., \& Carver, C. S. (2006). Extreme goal setting and vulnerability to mania among undiagnosed young adults. Cognitive Therapy and Research, 30, 377-395.

[16] Randles, D., Flett, G. L., Nash, K. A., McGregor, I. D., \& Hewitt, P. L. (2010). Dimensions of perfectionism, behavioral inhibition, and rumination. Personality and Individual Differences, 49, 83-87.

[17] Rasmussen, S. A., Elliott, M. A. \& O'Connor, R. C. (2012). Psychological distress and perfectionism in recent suicide attempters: The role of behavioural inhibition and activation. Personality and Individual Differences, 52(6), 680-685.

[18] Smillie LD, Jackson CJ. The appetitive motivation scale and other BAS measures in the prediction of approach and active avoidance. Personality and Individual Differences. 2005; 38(4):981-994.

[19] Stoeber, J., \& Corr, P. J. (2015). Perfectionism, personality and affective experiences: New insights from Reinforcement Sensitivity Theory. Personality and Individual Differences, 86, 354-359.

[20] Stoeber, J., \& Otto, K. (2006). Positive conceptions of perfectionism: Approaches, evidence, challenges. Personality and Social Psychology Review, 10, 295-319. doi: $10.1207 / \mathrm{s} 15327957$ pspr1004_2

[21] Stumpf, H. \& Parker, W. D. (2000). A hierarchical structural analysis of perfectionism and its relation to other personality characteristics. Personality and Individual Differences, 28(5), 837-852.

[22] Torrubia, R., Avila, C., Molto, J., \& Caseras, X. (2001). The sensitivity to punishment and sensitivity to reward questionnaire (SPSRQ) as a measure of Gray's anxiety and impulsivity dimensions. Personality and Individual Differences, 31, 837-862. doi: $10.1016 / \mathrm{S} 0191-$ 8869(00)00183-5

[23] Turner, L. A. \& Turner, P. E. (2011). The relation of behavioral inhibition and perceived parenting to maladaptive perfectionism in college students. Personality and Individual Differences, 50(6), 840-844. 